\title{
This is not a tile, just a digital remix
}

\author{
Elisa Antunes ${ }^{\dagger}$ \\ Centro de Investigação em Artes e \\ Comunicação \\ Universidade do Algarve \\ Faro, Portugal \\ Elisaantunese@gmail.com
}

\author{
José Bidarra \\ Universidade Aberta \\ Lisboa, Portugal \\ Centro de Investigação em Artes e \\ Comunicação \\ Universidade do Algarve \\ Faro, Portugal \\ Jose.Bidarra@uab.pt
}

\author{
Alexandra Gago da Câmara \\ Universidade Aberta \\ Lisboa, Portugal \\ Centro de História de Arte e \\ Investigação Artística \\ Universidade de Évora \\ Évora, Portugal \\ matgc@uab.pt
}

\begin{abstract}
This article intends to establish a transformative relationship between digital narratives and audiovisual content based on digital archives and the possible synergies with digital media art. It originates from aesthetic and functional experimentation, based on emerging digital technologies involving the manipulation and re-editing of images, - what we call "digital remix".

In this sense, a prototype was developed with augmented reality technology supported by the Aurasma application, consisting of overlays of a digital narrative about an educational video, dealing with the 18th century tiles of Palácio Ceia.
\end{abstract}

\section{RESUMO}

Este artigo pretende estabelecer uma relação transformadora entre narrativas digitais e conteúdos audiovisuais com base em arquivos digitais e as sinergias possíveis com a média-arte digital. Tem origem na experimentação estética e funcional, assente em tecnologias digitais emergentes implicando a manipulação e a re(edição) de imagens - o que designamos de "remix digital". Nesse sentido, foi desenvolvido um protótipo com a tecnologia de realidade aumentada suportada pela aplicação Aurasma, consistindo em overlays de uma narrativa digital sobre um vídeo educativo, tratando a azulejaria setecentista do Palácio Ceia.
Permission to make digital or hard copies of all or part of this work for personal or classroom use is granted without fee provided that copies are not made or distributed for profit or commercial advantage and that copies bear this notice and the full citation on the first page. Copyrights for components of this work owned by others than ACM must be honored. Abstracting with credit is permitted. To copy otherwise, or republish, to post on servers or to redistribute to lists, requires prior specific permission and/or a fee. Request permissions from Permissions@acm.org.

ARTECH 2019, October 23-25, 2019, Braga, Portugal (c) 2019 Association for Computing Machinery. ACM ISBN 978-1-4503-7250-3/19/10..\$15.00 https://doi.org/10.1145/3359852.3359917

\section{CCS CONCEPTS}

- Information systems $\rightarrow$ Multimedia content creation • Applied computing $\rightarrow$ Media Arts -Applied computing $\rightarrow$ Digital libraries and archives

\section{KEYWORDS}

Média-arte digital, narrativas digitais, realidade aumentada, remix digital

\section{ACM Reference format:}

Elisa Antunes, José Bidarra and Alexandra Gago da Câmara. 2019. This is not a tile, just a digital remix. 2019. In Proceedings of Artech 2019, 9th International Conference on Digital and Interactive Arts (Artech 2019), Braga, Portugal, 4 pages. https://doi.org/10.1145/3359852.3359917

\section{Introdução}

Os novos media digitais implicam novos contornos para o diálogo entre o homem e a história. Segundo Wolske [1], o consumo de produtos televisivos, ainda hoje preponderante, está a perder para um acelerado avanço das audiências dos ambientes online. Os fatores principais deste novo contexto são essencialmente dois. Em primeiro lugar, a capacidade de ver os conteúdos online a qualquer momento e em qualquer lugar e, em segundo lugar, a tendência para o visionamento de conteúdos mais curtos, com pouca complexidade e pouco profissionalismo.

O consumo em dispositivos móveis, por exemplo, altera a experiência audiovisual, pois os ecrãs pequenos prejudicam a leitura de pequenos detalhes. As imagens são apresentadas com pouca nitidez e reduzida qualidade fotográfica, diminuindo o seu impacto e fruição estética. Por outro lado, existe uma capacidade de interação com os conteúdos digitais que permite um maior envolvimento do leitor/utilizador. Assim, neste estudo, definimos um novo contexto para a comunicação multimédia e audiovisual. $\mathrm{O}$ contexto do estudo baseou-se na linguagem da média-arte digital e a sua articulação com a linguagem audiovisual. Para este fim, estabelecemos algumas considerações sobre as dinâmicas que enquadram hoje as narrativas digitais, procurando compreender a 
sua complexidade quando enquadradas em tecnologias emergentes. Por fim, damos como exemplo uma instalação de arte digital usando uma forma simples de realidade aumentada.

\section{Narrativas Digitais}

O conceito de storytelling tem sido amplamente discutido, principalmente na associação com as novas tecnologias digitais. No entanto, contar histórias é uma atividade recorrente da humanidade. Desde a Antiguidade, na tradição oral e na escrita, acompanhamos o desenrolar de narrativas. É interessante perceber como as narrativas, nos tempos atuais, ocupam os novos formatos. Tomam um carácter fluido, uma espécie de propagação, que permite estabelecer um diálogo importante com o público.

Assim, hoje assiste-se ao renascimento global de um modelo designado no mundo anglo-saxónico como "digital storytelling", que poderia ser traduzido como "narrativas digitais", e neste caso não se trata de uma moda; as histórias e narrativas têm sido usadas no ensino e na aprendizagem desde há muito, mas, também em áreas como a gestão, a psicologia ou a saúde. As histórias ajudamnos a compreender a experiência individual e a criar uma perceção do mundo que nos rodeia. As histórias também ajudam a construir ligações com o conhecimento adquirido e a melhorar a memória, como resultado, as boas histórias são melhor lembradas pelos alunos do que as aulas regulares. Por outro lado, dado o papel central da narrativa no nosso quotidiano e a explosão tecnológica durante estas últimas décadas, não é surpreendente encontrar narrativas digitais como estratégias pedagógicas em muitos cursos universitários, tal como são essenciais para o teatro, os filmes e os jogos.

Mas o que são "narrativas digitais"? Essencialmente, referimo-nos a artefactos digitais que incluem: uma narração convincente de uma história; um contexto significativo para a compreensão da história; texto, imagens e gráficos que capturam ou expandem as emoções encontradas na narrativa; voz, música e efeitos sonoros que reforçam temas; e mecanismos que convidam à reflexão e envolvimento do público-alvo.

As narrativas referem-se a uma série de eventos que aconteceram (no passado, como uma memória), estão a acontecer (no presente) ou vão acontecer (no futuro). Por seu turno, contar histórias implica integrar quatro elementos clássicos que são ainda válidos na era digital, ou seja: uma trama; um narrador; um contexto; as personagens. De uma forma simples, poderíamos dizer que uma narrativa digital é aquela narrativa que está suportada em meios digitais. Atualmente esta expressão abrange um conjunto grande de projetos e de produtos que podem incluir: livros de recortes e fotografias (scrapbooks), portfólios, peças de radiodifusão (podcasts), projetos multimédia e vídeos. As ferramentas digitais postas ao alcance de qualquer um, como parte do software livre da Web 2.0, abriram um novo mundo de aplicações para utilização em boas práticas educativas. Um bom exemplo é o uso da timeline do Facebook para narrar uma cronologia (viagem, experiência, investigação, evento).

Para Ursyn [2], existe evidência de que os mitos, lendas e contos tradicionais estão a ser transportados para ambientes online. $\mathrm{O}$ evidente potencial dos meios de comunicação atuais baseados na internet, fez emergir algumas caracterizações relativamente à forma como são estabelecidas as interações entre pessoas e plataformas digitais. Esses contactos entre formas tradicionais da imagem, do som e do verbo, ganham contornos mais interativos e dinâmicos que permitem uma visualização muito intensa dos objetos. Essa interação é operada tanto por quem produz como por quem recebe as mensagens. Assim, esta nova dinâmica muda drasticamente o antigo status da comunicação que deixava emissores, recetores e produtores isolados e atuando dentro do processo comunicativo de formas diferenciadas, como acontece na televisão.

Nesse sentido, os próprios meios de comunicação começaram a perceber o potencial interativo da internet e estruturaram novos caminhos em rede. Surge assim um complexo fluxo de interações, descritas de forma clara por Jenkins [3] no seu livro Cultura de Convergência. Este autor define vários conceitos novos para estes contextos digitais, estabelecendo a ideia de narrativa transmedia, que se refere a esse cenário de propagação de conteúdos através de diferentes canais. A narrativa transmedia não é simplesmente a apresentação de conteúdos em diferentes meios de comunicação, mas sim uma transferência narrativa entre conteúdos projetados para diferentes plataformas de comunicação [4].

É importante perceber que, nestas transferências, as narrativas moldam-se e enquadram-se em diferentes formatos e combinações. Segundo Ursyn [2], o storytelling visual introduz uma quarta dimensão, pois possibilita criar um novo modelo temporal e espacial para seguir as peripécias das personagens e da sua ação. As personagens, no ecrã do computador ou do smartphone, transmitem emoções e informações que são recebidas pela audiência. Neste contexto, ganha importância a "média-arte digital", que desenvolve técnicas e poéticas próprias, a fim de construir possíveis narrativas visuais em ambientes de interação. Assim, Alexander [5] leva mais longe o conceito de digital storytelling, nomeadamente, o autor coloca uma questão importante: como pode uma história existir fora do tempo? É verdade que as narrativas lineares são importantes na construção de aspetos de nossa História, Artes, Literatura, etc., até mesmo em produtos como telenovelas, filmes e vídeos que tendem para a construção ordenada de narrativas. Mas tudo isto mudou com os novos artefactos digitais, hiperligados, em rede, e em constante mutação por via de algoritmos, sensores, atuadores.

\section{Criação de um Artefacto}

Este projeto seguiu um processo iterativo envolvendo componentes conceptuais e técnicas. Nomeadamente, seguiu as várias fases do processo de criação de um artefacto digital genérico: i) desenho da mensagem; ii) meditação estética; iii) e desenvolvimento do artefacto. Na instalação "Isto é um Azulejo? Is this a Tile?" procedemos à elaboração do conceito e da narrativa, integrados numa experiência de apreensão estética, sem descurar a inovação tecnológica e a implementação técnica do artefacto. Somente o planeamento da instalação ficou aprazada para a data da exposição, que decorreria meses mais tarde.

Os ciclos de reflexão/reverberação decorreram durante todo o processo de criação do artefacto, desde a visão inicial - teoria - com o aparecimento da ideia e a visualização do que poderia ser o artefacto; depois a utilização de tecnologias e materiais - praxis neste caso com a instalação da aplicação Aurasma em smartphone e em tablet, incluindo a parametrização dos marcadores com as imagens pretendidas, o carregamento do vídeo pretendido e a escolha da imagem destinada a ser visualizada como marcador (Figura 1); finalmente, a materialização do artefacto - poesis - com a experiência a decorrer, com o visionamento do vídeo "Brilhos e Reflexos na Azulejaria de Setecentos: o Palácio Ceia”, (re)editado 
This is not a tile, just a digital remix

e a possibilidade de interação em Realidade Aumentada (via Aurasma).

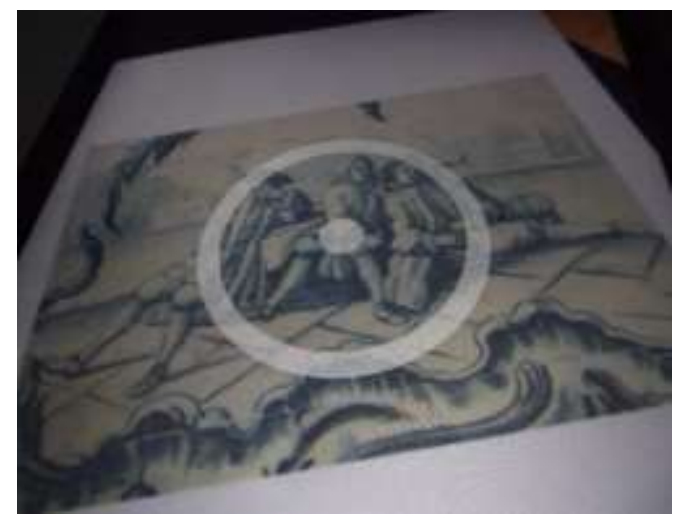

Figura 1: Marcador RA

A (re)edição do vídeo foi efetuada através de remix digital, mantendo a mensagem original, mas com alterações do conteúdo, nomeadamente na forma, ou seja, nas imagens. Procurou-se a utilização de planos fixos, sem movimentos, com enquadramentos médios, evitando os planos muito gerais, com falta de leitura e que perdessem muita informação e, no caso oposto, - planos muito próximos, - que evidenciassem perda de qualidade e resolução da imagem. Com o remix e o corte de planos, o vídeo ficou com a duração reduzida, mais conveniente para a visualização em dispositivos móveis e através de aplicações. Foi ainda tomada em consideração a preservação do registo áudio original, independentemente do remix digital, salvaguardando os conteúdos pedagógico e científico.

Em termos narrativos, houve que considerar as características diferenciadoras da estética computacional que alteram as do vídeo original, nomeadamente, a tecnologia, o hibridismo, a aleatoriedade, a virtualidade e a imersão, no sentido de aperfeiçoar o artefacto. Assim, o processo de reflexão - experimentação materialização foi constante e efetuado de forma iterativa. Por outro lado, a meditação estética enquanto ciclo de reflexão intensificou o sentido da obra, clarificando a necessidade do projeto evoluir e desenvolver-se através de uma análise criteriosa. Neste sentido, consideramos que as práticas de experimentação e reformulação, em contexto metodológico de a/r/tografia [6], transformam o processo de criação artística em investigação, em arte e tecnologia, proporcionando a emergência de novos discursos, aplicações estéticas e artísticas, significações e sentidos, interligando arte, investigação e aprendizagem, em convergência com os conceitos de Theoria e Métissage [7]. A aprendizagem adquirida no processo de reflexão - experimentação materialização evidenciou a possibilidade de criação de novos projetos futuros, com a utilização de outros vídeos de arquivo, especificamente, para utilização em contexto educativo.

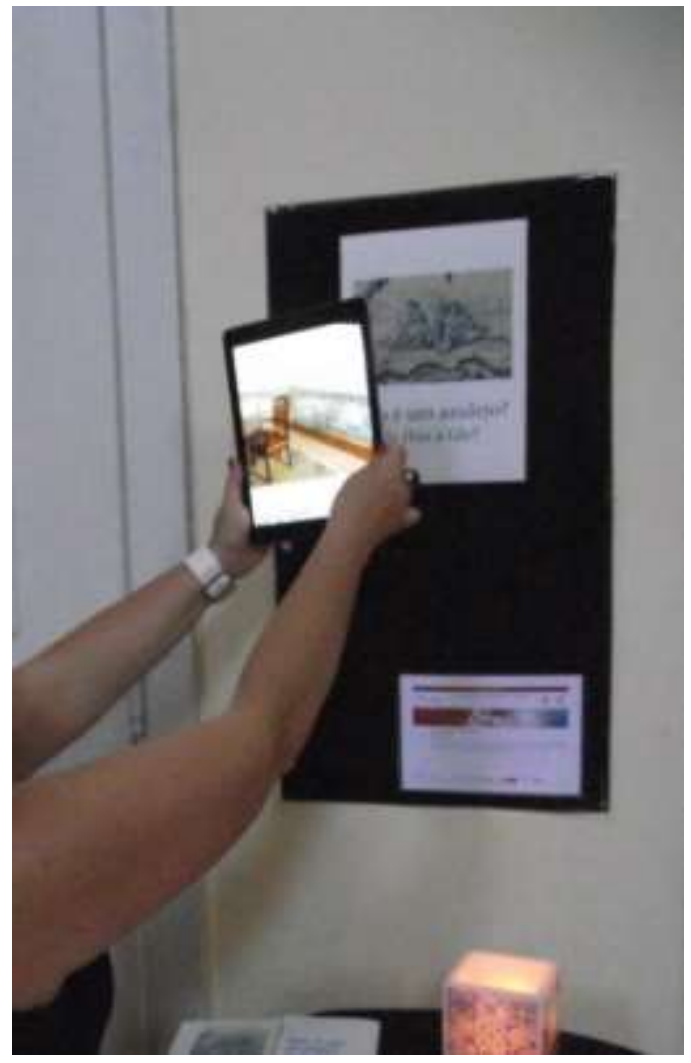

Figura 2: Exposição da Instalação

A instalação foi exposta durante um Retiro Doutoral, em Lisboa, Alfama, de 18 a 23 de julho de 2016 (Figura 2), integrado no Festival da Paratissima, tendo obtido aceitação muito satisfatória por parte dos visitantes da exposição. A curiosidade dos visitantes pelo tema dos azulejos portugueses foi evidente, e o uso da Realidade Aumentada (RA) através da aplicação Aurasma foi objeto de comentários positivos por visitantes de diferentes nacionalidades (inglesa, francesa e espanhola). Desta forma, os dois objetivos principais deste projeto foram plenamente atingidos: o remix digital resultante da (re)utilização de materiais audiovisuais de arquivo em novos produtos digitais e a divulgação do património de azulejaria do Palácio Ceia, em Lisboa.

\section{Conclusão}

Na perspetiva do desenvolvimento de um remix audiovisual com capacidade interativa, a maior parte dos objetivos foram atingidos. Em termos de avaliação heurística, os propósitos artísticos foram atingidos, as escolhas feitas revelaram-se acertadas e a reverberação junto do público foi encorajadora.

Refletindo sobre todos estes processos, podemos reconhecer as inúmeras potencialidades que a média-arte digital possibilita ao estimular experiências interativas, permitindo a persistência da memória como verificamos no contexto de "digital storytelling", e a existência de eventos significativos ligados a um artefacto digital, neste caso (re)utilizando material audiovisual de arquivo num novo produto digital interativo. 
O remix digital surge, assim, como um fator potenciador de novas leituras e indissociável da partilha de imagens de forma colaborativa convocando, a ideia de Priscila Arantes [8] de que "ao partilharmos imagens construímos um bem comum que nos une”.

\section{REFERÊNCIAS}

[1] Julian Wolske. 2014. Interactive storytelling and experiental learning: the prospect of "vertical narrativity". in F. M. M. Neto (Ed.) Technology Platform Innovations and Forthcoming Trends in Ubiquitous Learning (pp. 194-214). Hershey PA, USA: IGI Global. doi: 10.4018/978-1-4666-4542-4.ch011

[2] Anna Ursyn. 2014. Visual and verbal storytelling, in A. Ursyn (Ed.) Computational Solutions for Knowledge, Art, and Entertainment: Information Exchange Beyond Text (pp. 429-455). Hershey PA, USA: IGI Global. doi: 10.4018/978-1-4666-4627-8.ch022

[3] Henry Jenkins. 2008. Cultura da Convergência. São Paulo: Aleph

[4] Patricia Rodrigues e José Bidarra. 2016. Transmedia storytelling as an educational strategy: a prototype for learning English as a second language, International Journal of Creative Interfaces and Computer Graphics (IJCICG): Special Issue on the ARTECH 2015 International Conference, 7(2), 55-66.

[5] Bryan Alexander. 2011. The new digital storytelling: creating narratives with new medias. Santa Barbara: Praeger.

[6] Rita Irwin et al. 2006. The Rhizomatic Relations of $\mathrm{A} / \mathrm{r} / \mathrm{tography}$, Southern Illinois University Carbondale OpenSIUC, Studies in Art Education, National Art Education Association, A Journal of Issues and Research 2006, 48(1), 70-88

[7] Adérito Marcos. 2001. Instanciando mecanismos de a/r/tografia no processo de criação em arte digital/computacional, $23^{\circ}$ Encontro da Associação de Professores de Expressão e Comunicação Visual (APECV), Escola Superior de Educação, Bragança.

[8] Priscila Arantes. 2018. Memória, Arquivo e Curadoria na Cultura Digital. In Proceedings of 1st International Conference on Transdisciplinary Studies in Arts, Technology and Society, ARTeFACTo 2018 ISBN: 978-989-99370-7-9 